\title{
Erratum: Whole-genome sequence-based analysis of thyroid function
}

Peter N. Taylor, Eleonora Porcu, Shelby Chew, Purdey J. Campbell, Michela Traglia, Suzanne J. Brown, Benjamin H. Mullin, Hashem A. Shihab, Josine Min, Klaudia Walter, Yasin Memari, Jie Huang, Michael R. Barnes, John P. Beilby, Pimphen Charoen, Petr Danecek, Frank Dudbridge, Vincenzo Forgetta, Celia Greenwood, Elin Grundberg, Andrew D. Johnson, Jennie Hui, Ee M. Lim, Shane McCarthy, Dawn Muddyman, Vijay Panicker, John R.B. Perry, Jordana T. Bell, Wei Yuan, Caroline Relton, Tom Gaunt, David Schlessinger, Goncalo Abecasis, Francesco Cucca, Gabriela L. Surdulescu, Wolfram Woltersdorf, Eleftheria Zeggini, Hou-Feng Zheng, Daniela Toniolo, Colin M. Dayan, Silvia Naitza, John P. Walsh, Tim Spector, George Davey Smith, Richard Durbin, J. Brent Richards, Serena Sanna, Nicole Soranzo, Nicholas J. Timpson, Scott G. Wilson \& the UK10K Consortium

Nature Communications 6:5681 doi: 10.1038/ncomms6681 (2015); Published 6 Mar 2015; Updated 20 May 2015

The original version of this Article noted incorrect affiliations for members of the UK10K Consortium, and contained typographical errors in the spelling of the UK10K Consortium and consortium members Valentina Iotchkova and Michael Quail. In addition, the author J. Brent Richards was incorrectly duplicated in the list of consortium members as Brent Richards. These errors have now been corrected in the PDF and HTML versions of this article. 